tools must be, until the desired proofs would be found.

References $[15]$ and $[16]$ were called to the author's attention by K. A. Hirsch.

Convair Astronautics

San Diego, California

1. H. E. SAlzER, "Tetrahedral numbers," $M T A C$, v. 1, 1943, p. 95.

2. "Table of first two hundred squares expressed as a sum of four tetrahedral numbers," Amer. Math. Soc., Bull., v. 49, 1943 , p. 688.

3. - , "New tables and facts involving sums of four tetrahedral numbers," Amer. Math. Soc., Bull., v. 50, 1944, p. 55.

4. "On numbers expressible as the sum of four tetrahedral numbers," London Math. Soc., Jn., v. 20,1945 , p. 3-4. This note includes a table of the exceptional numbers $\leq 1000$ (erratum: omission of 107). That table is continued up to 2000 in $M T A C$, v. 3, 1949, p. $423-424$.

5. - "Further empirical results on tetrahedral numbers," Amer. Math. Soc., Bull., v. 52, 1946, p. 420.

6. . "An 'empirical theorem' which is true for the first 618 cases, but fails in the 619th," Amer. Math. Soc., Bull., v. 53, 1947, p. 908 (errata on p. 1196).

7. "Table expressing every square up to one million as a sum of four non-negative tetrahedral numbers," Amer. Math. Soc., Bull., v. 54, 1948, p. 830.

8. - "Representation table for squares as sums of four tetrahedral numbers," $M T A C, \mathrm{v} .3$, 1948 , p. 316.

9. of a device for mass computation," Amer. Math. Soc., Bull., v. 55, 1949, p. 41.

Empirical Theorems of Other Authors:

10. F. Pollock, "On the extension of the principle of Fermat's Theorem of the polygonal numbers to the higher orders of series whose ultimate differences are constant. With a new theorem proposed, applicable to all the orders," Roy. Soc. London, Proc., S A, v. 5, 1850, p. 922-924.

11. H. W. Richmond, "Notes on a problem of the 'Waring' type," London Math. Soc., Jn., v. 19,1944 , p. $38-41$.

Mathematical Papers:

12. L. E. Dickson, History of the Theory of Numbers, v. 2, Diophantine Analysis, Carnegie Institute of Washington, publication no. 256, v. II (reprinted by G. E. Stechert, N. Y., 1934), p. iv-v and Chapter I, especially p. 4, 7, 22-23, 25, 39.

13. L. E. Dickson, Modern Elementary Theory of Numbers, University of Chicago Press, Chicago, 1939, Chap. VII, "Sums of nine values of a cubic function," p. 130-146. This contains the proof of the theorem that every integer is the sum of nine tetrahedrals, with further references to the most advanced work up to that time, that of R. D. JAMES and L. K. HUA on the theorem that every sufficiently large integer is the sum of eight tetrahedrals.

14. G. L. WATSON, "Sums of eight values of a cubic polynomial," London Math. Soc., Jn., v. 27,1952 , p. $217-224$. This includes the proof of the theorem that every integer is the sum of eight tetrahedrals.

15. L. K. HUA, "Sur le problème de Waring relatif à un polynome du troisième degré," Comptes Rendus, Academie des Sciences, Paris, v. 210, 1940, p. 650-652.

16. L. K. HUA, "On Waring's problem with cubic polynomial summands," Sci. Report Nat. Tsing Hua Univ., (A) v. 4, 1940, p. 55-83.

\title{
GROUPS OF PRIMES HAVING MAXIMUM DENSITY
}

\section{By John Leech}

The following lists give groups of six or more primes which minimize the difference between first and last, the lists being complete for the range 50 to 100 00000. Four numbers out of nine can be prime, such as 191, 193, 197, 199. There are 897 such groups of four in the range. Five numbers out of thirteen can be prime; there are 318 such groups in the range. Six numbers out of seventeen can be prime, such as $97,101,103,107,109,113$; there are seventeen such groups in the range, centered on:

Received February 7, 1955. Due to misfiling in the $M T A C$ office, this paper is appearing later than was scheduled; see $M T A C$, Review 110, v. 11,1957, p. 274 . Some of the results have meanwhile appeared in "On a generalization of the prime pair problem," by Herschel F. Smith, $M T A C$, v. 11,1957, p. 274. 


$\begin{array}{rrrr}105 & 1091265 & 2839935 & 6503595 \\ 16065 & 1615845 & 3243345 & 7187775 \\ 19425 & 1954365 & 3400215 & 7641375 \\ 43785 & 2822715 & 6005895 & 8062005\end{array}$

8741145

Seven numbers out of 21 can be prime, eight out of 27 and nine out of 31 . These all occur and are listed below. It is possible for ten numbers out of 33 to be prime and eleven out of 37 , but these do not occur in the range; included in the list are such groups of nine out of 33 and ten out of up to 37 as occur in the range.

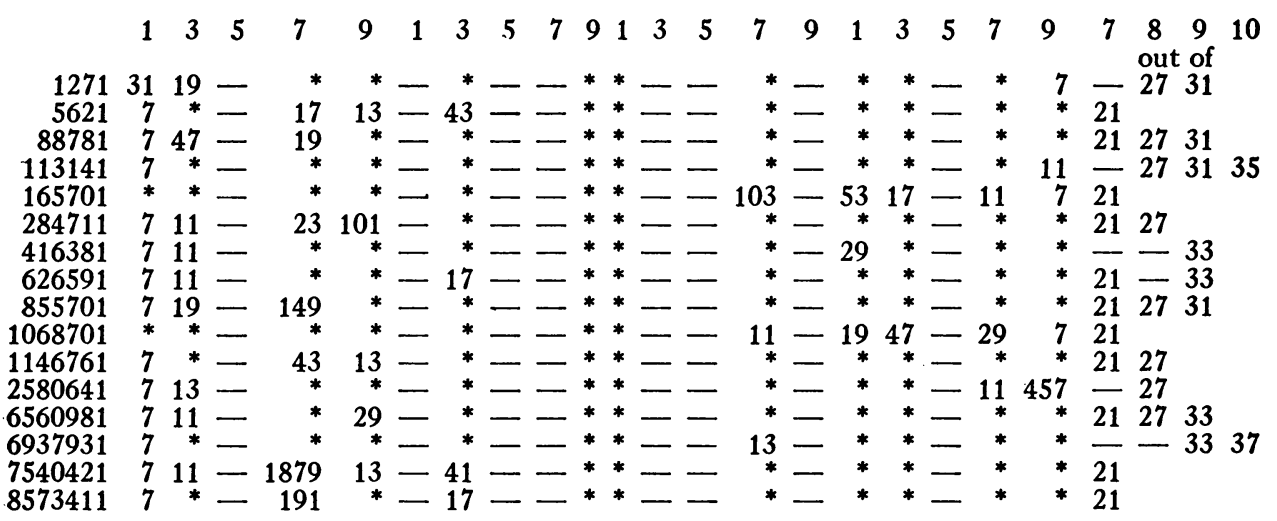

In each line, the number in the left hand column is the first number for the line, an asterisk indicates a prime, a dash a multiple of 3 or 5 and a number the least factor of any other composite. There are thus seen to be eleven groups of seven primes in 21 numbers, eight of eight primes in 27 , five of nine primes in 31 (two in the line beginning with 113141) and four more of nine in 33, and one group of ten in 35 and one of ten in 37 . The range 1 to 50 is excluded as being altogether exceptional. A list of the groups of four and five has been deposited in the $U M T$ file of $M T A C$ (see Review 110, $M T A C$, v. 11, 1957, p. 274).

King's College

Cambridge, England

\section{REVIEWS AND DESCRIPTIONS OF TABLES AND BOOKS}

$60[\mathrm{~A}, \mathrm{~B}, \mathrm{C}, \mathrm{D}, \mathrm{H}, \mathrm{I}, \mathrm{K}, \mathrm{L}, \mathrm{M}, \mathrm{P}, \mathrm{R}, \mathrm{V}, \mathrm{X}, \mathrm{Z}]$.-George E. Forsythe, Bibliography of Russian Mathematics Books, Chelsea Publishing Co., New York, 1956, 106 p., $20 \mathrm{~cm}$. Price $\$ 3.95$.

Professor Forsythe precedes his very useful bibliography of Russian mathematical books with an informative introduction which contains, among others, a complete review of the book. To quote Prof. Forsythe:

"The subject matter of the books listed is mathematics, pure and applied, including tables beyond the most elementary, but excluding descriptive geometry. There are a few titles on quantum mechanics and other branches of mathematical physics, and more on mechanics, mathematical machines and nomography, but 